\title{
Invisibilité et transparence
}

\author{
Boris Gralak ${ }^{1}$ et Sébastien Guenneau ${ }^{2}$
}

1.Aix-Marseille Université, CNRS, Centrale Marseille, Institut Fresnel, Marseille, France

2.UMI 2004 Abraham de Moivre, CNRS-Imperial, Imperial College London, Londres, Royaume-Uni

Résumé : L'invisibilité et la transparence sont des notions associées à des objets fascinants de la littérature fantastique et l'imaginaire; elles sont appréhendées à travers le prisme des ondes lumineuses - la lumière - dont elles adoptent le langage. Ces notions ne sont pas moins fascinantes dans le monde des ondes radioélectriques, acoustiques et mécaniques où l'invention des métamatériaux laisse entrevoir des applications en photonique et génie civil grâce aux dernières avancées scientifiques et techniques.

mots-clés : invisibilité, transparence, métamatériaux, lumière, vagues, séismes, ondes

Introduction : L'invisibilité est un thème récurrent de la mythologie avec notamment la Kunée d'Hadès, un casque qui rend son porteur invisible, offert par les cyclopes au dieu grec des Enfers. L'invisibilité apparaît au détour de la littérature fantastique avec l'anneau à détruire de la trilogie de John Ronald Reuel Tolkien, qui passé au doigt du Hobbit Frodon Sacquet, le rend invisible aux yeux des vivants, mais pas des neufs cavaliers noirs, spectres de l'anneau. L'invisibilité est donc une notion qui se définit par rapport à un observateur donné (en l'occurrence les vivants ou les spectres). La relativité de la notion d'invisibilité transparait notamment dans la célèbre réplique du renard au petit prince d'Antoine de Saint-Exupéry « L'essentiel est invisible aux yeux, on ne voit bien qu'avec le cœur ».

Il en est de même en physique des ondes : des capes d'invisibilité ont été fabriquées, telle la cape d'invisibilité pour les vagues (voir la Figure 1) qui sont bien visibles à l'œil humain : l'invisibilité est réalisée ici pour les ondes à la surface de l'eau, qui ne perçoivent pas la présence de la cape, mais pas pour les ondes lumineuses.

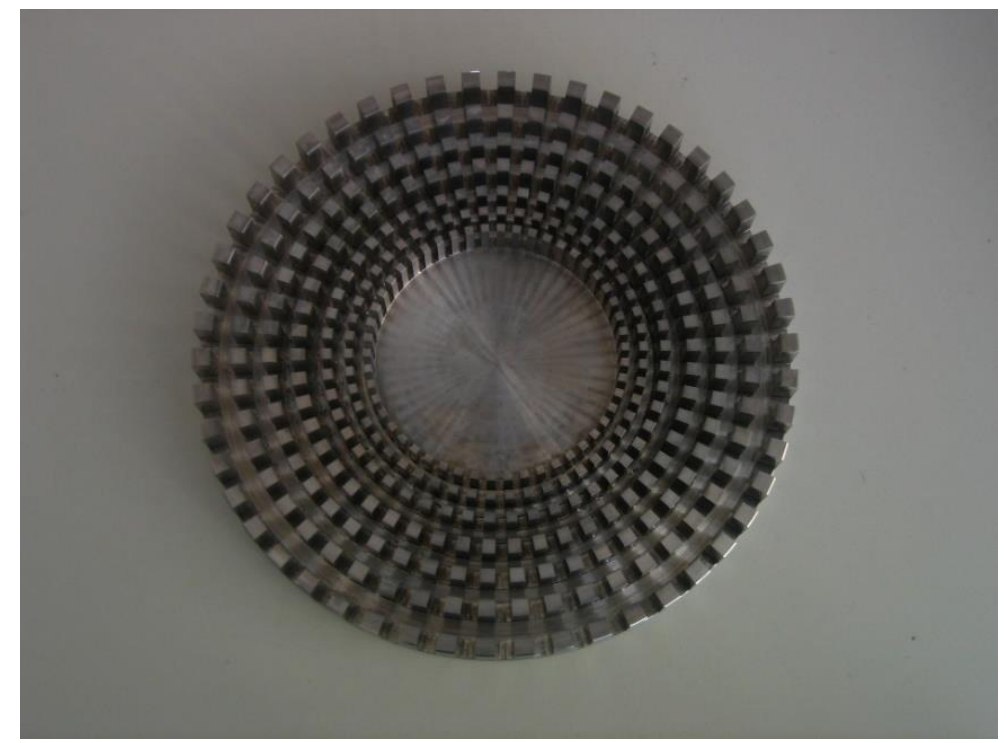

Figure 1 : Photographie de la cape d'invisibilité pour les vagues en vue de dessus; Ce métamatériau de 20 centimètres de diamètre est constitué de 7 rangées concentriques de petits pylônes de 1 centimètre de haut. Quand les vagues pénètrent dans la cape, elles privilégient un trajet contournant la zone au centre du dispositif qui est donc protégée des vagues. (crédit photo: Photothèque CNRS, Institut Fresnel). 


\section{Obscurité, transparence et invisibilité}

Revenons à l'invisibilité proprement dite et donc aux ondes lumineuses, c'est-à-dire les ondes électromagnétiques dont les longueurs d'onde dans le vide varient de 400 à 750 nanomètres ${ }^{\text {(note de bas }}$ de page : un nanomètre est un milliardième de mètre) : que signifie précisément "voir un objet " ? On ne voit pas directement un objet comme on pourrait le ressentir au toucher, mais notre œil perçoit des ondes lumineuses qui proviennent d'une source de lumière - le soleil, une ampoule lumineuse - et qui sont renvoyées par l'objet jusqu'à notre pupille. Pour rendre un objet invisible, la première solution consiste à supprimer la source de lumière : tous les objets sont invisibles dans l'obscurité totale car aucune onde lumineuse $n$ 'atteindra ces objets. Une deuxième solution est de supprimer les ondes lumineuses qui sont renvoyées par l'objet en rendant l'objet transparent: une surface vitrée peut sembler parfaitement transparente et invisible à l'œil nu. Un autre matériau remarquablement transparent pour les ondes lumineuses est l'eau liquide, et ce n'est pas un hasard ! En effet, la vie est apparue et s'est développée dans ce solvant unique que constitue l'eau liquide et l'évolution a permis aux êtres vivants de développer des organes sensibles aux ondes électromagnétiques capables de pénétrer assez profondément dans l'eau, les ondes lumineuses. La lumière peut pénétrer dans l'eau liquide de quelques mètres pour le rouge jusqu'à plusieurs dizaines de mètres pour le bleu, cette plus grande transparence pour le bleu expliquant la couleur de la mer en profondeur. Pour se rendre compte de la transparence remarquable de l'eau liquide pour la lumière, il faut imaginer un téléphone portable ou un poste de radio pouvant capter du signal ou des ondes radioélectriques à plus de 1000 kilomètres de profondeur.

Les deux solutions que nous avons tous à portée de main pour approcher l'invisibilité sont donc l'obscurité et la transparence. Mais la véritable invisibilité, celle qui est présente dans la littérature fantastique et de science-fiction, doit permettre de rendre invisible un objet non transparent en plein jour, c'est-à-dire en l'absence d'obscurité. Pour être invisible, un objet non transparent doit d'abord ne pas renvoyer de lumière et donc être protégé des ondes lumineuses : la solution consiste donc à entourer l'objet d'une cape pour le placer dans l'obscurité, de la même façon que l'on protège des vagues - les ondes à la surface de la mer - un port et les bateaux qui s'y trouvent avec une digue. La cape joue donc le rôle d'une boite noire à l'intérieur de laquelle c'est l'obscurité totale. Si on ne voit plus l'objet qui se trouve caché dans la boîte noire, on voit maintenant la cape sauf si cette dernière est justement... transparente ! Si la cape est transparente alors elle est invisible, et si la cape protège l'objet en le plaçant dans l'obscurité alors l'objet est aussi invisible.

Le secret de la cape d'invisibilité réside donc dans la capacité de créer de l'obscurité avec une cape transparente, c'est-à-dire de fabriquer une boîte noire qui soit aussi transparente. Jusqu'au début du XXIème siècle, cette éventualité est restée cantonnée au monde de l'imaginaire et du fantastique. La raison est que les matériaux dont nous disposons dans la nature sont transparents, absorbants ou réfléchissants, ou un peu des trois à la fois. L'eau liquide et le verre sont remarquablement transparents. Un objet parfaitement noir va absorber toute la lumière et l'énergie des ondes lumineuses pour la convertir en chaleur, c'est pour cela qu'il chauffe quand il est exposé au soleil. Les métaux sont particulièrement réfléchissants ou brillants et c'est grâce à l'aluminium qui réfléchit toutes les ondes lumineuses que nous pouvons voir notre image dans un miroir. De nombreux métaux et matériaux sont à la fois réfléchissants et absorbants : l'or absorbe le rouge, le bleu et le vert et réfléchit le jaune pour donner cette apparence jaune brillant si fascinante ; une couche de peinture bleue absorbe toutes les couleurs sauf le bleu que nous percevons. Si émerveillantes soient ces possibilités offertes par les matériaux naturels, elles ne permettent pas de concevoir une cape à la fois transparente et totalement hermétique à la lumière. Mais la découverte des métamatériaux au tournant de ce siècle par le physicien britannique Sir John Pendry aura permis de faire passer 
l'invisibilité de l'imaginaire à une thématique scientifique qui fédère sur la scène internationale des physiciens, des ingénieurs, des mathématiciens et des numériciens.

Comment un métamatériau peut-il permettre cette prouesse d'être à la fois transparent et totalement réfléchissant pour isoler un objet des ondes comme une digue protégeant un bateau des vagues ? Nous pouvons appréhender de nouvelles possibilités en revenant vers ce matériau essentiel que constitue l'eau liquide. Si l'eau liquide peut être qualifiée de transparente, la réalité est plus complexe. Une expérience assez courante consiste à se retrouver ébloui par la surface de l'eau quand le soleil brille : en effet, sous certains angles, les ondes lumineuses ne pénètrent plus dans l'eau liquide mais sont au contraire réfléchies. Sur un bateau au milieu d'un lac ou d'une mer calme, nous serons immanquablement éblouis par la lumière du soleil si celui-ci n'est pas trop haut dans le ciel, par exemple le matin ou le soir. Cela signifie qu'un même matériau peut être transparent ou totalement réfléchissant comme un miroir suivant l'angle d'attaque des ondes lumineuses. Une expérience semblable peut être réalisée avec une surface vitrée. Nous pouvons alors imaginer qu'en jonglant avec la surface d'un matériau transparent il peut être possible de combiner les propriétés de transparence de l'eau liquide et de réflexion d'un miroir en argent.

Les métamatériaux exploitent une structuration conçue dans un but précis en combinant différentes propriétés optiques par un jeu subtile d'interfaces. Cette conception révolutionnaire repose sur des connaissances très pointues développées depuis des décennies en mathématiques appliquées avec la théorie de l'homogénéisation et des matériaux composites et en physique avec la conception de composants résonants et de propriétés effectives. Les métamatériaux permettent ainsi de concevoir de nouveaux composants comme les lentilles plates et les capes d'invisibilité.

Réalisations de capes d'invisibilité pour différents types d'ondes : Une cape d'invisibilité a été réalisée par une équipe anglo-américaine en 2006 pour des micro-ondes qui font partie du spectre des ondes électromagnétiques invisibles à l'œil humain (mais tout comme la cape à vagues en Figure 1, cette cape et le cylindre de cuivre qu'elle soustrait aux micro-ondes sont bien visibles pour un observateur humain) ; une cape a été réalisée par une équipe allemande en 2012 pour des ondes mécaniques dans les plaques, et un prototype de cape sismique a même été testé par l'entreprise Ménard dans la région lyonnaise en 2012. Néanmoins, ces capes d'invisibilité ne permettent pas de soustraire un objet à notre regard, la cape d'invisibilité portée par le personnage Harry Potter de la romancière Joanne Rowling semble encore bien loin. D'ailleurs de quoi sont faites ces capes d'invisibilité ?

Les capes d'invisibilité sont des matériaux sortis des laboratoires universitaires au cours des vingt dernières années qui possèdent des propriétés électromagnétiques, acoustiques, mécaniques et thermiques que l'on ne rencontre pas dans la nature (" méta » signifie " au-delà » en grec). A titre d'exemple, reprenons la cape à vagues (voir figure 1). Que font donc les vagues qui passent à travers ce métamatériau ? Elles contournent le centre de la cape à la manière de l'eau autour du rocher dans le cours d'une rivière. En aval, le courant est identique à l'amont. L'objet, derrière son bouclier, est comme transparent : le sillage derrière l'objet a disparu! Mais il y a plus : les simulations numériques et expériences en bassin démontrent que les vagues qui se propagent dans la cape se reforment derrière la cape sans déphasage par rapport à une vague se propageant à la surface de l'eau en l'absence d'obstacle. Or le chemin qu'elles parcourent à travers la cape est plus long que celui qu'elles parcourent en eau libre (id est sans obstacle). Cela signifie que la cape accélère les vagues qui la traversent, de telle sorte qu'elles rattrapent celles qui se propagent en ligne droite à côté de la cape !

Un paradoxe optique de la cape d'invisibilité pour la lumière du visible : Ce qui paraît assez naturel pour les ondes hydrodynamiques dans la cape à vagues, devient plus problématique pour les ondes électromagnétiques : ces dernières suivent dans une cape d'invisibilité pour la lumière un chemin 
optique plus long que celles qui passeraient à travers un objet s'il était transparent, mais elles rattrapent leur retard. C'est un paradoxe optique, car la lumière ne peut se propager plus vite que dans le vide où, d'après la théorie de la relativité d'Einstein, elle ne peut dépasser 299792458 mètres par seconde. Que se passe-t-il donc dans cette cape qui paraît bien magique ? Cette cape est constituée $d^{\prime}$ 'un matériau très hétérogène (son indice de réfraction n'est pas constant mais il varie en chaque point de la cape), de sorte qu'au niveau microscopique des phénomènes d'interférence complexes sont mis en jeu dans la cape. Au niveau macroscopique, tout se passe comme si la cape était constituée d'un matériau homogène aux propriétés exotiques, défiant les lois de la physique. L'indice de réfraction dit effectif de cette cape peut prendre des valeurs inférieures à 1 (voire même négatives) quand leur fréquence est en résonance avec les petits éléments qui constituent l'ossature de la cape. Dès lors, une vitesse de phase effective de la lumière supérieure à 299792458 mètres par seconde devient possible dans la cape d'invisibilité, tout au moins pour une onde lumineuse dont la fréquence est proche de la fréquence de résonance des éléments de la cape. En pratique, l'absorption et l'impossibilité de travailler à une seule longueur d'onde pour la lumière évitent tout paradoxe ou bizarrerie : le spectre visible est constitué d'un continuum de fréquences (c'est-à-dire de couleurs), et non pas d'une seule fréquence. L'effet magique d'une cape d'invisibilité en métamatériau ne se produit qu'autour d'une fréquence et les métaux constituant les résonateurs sont fortement dissipatifs aux longueurs d'ondes optiques[ref1]. Il y aura bien un retard mesurable induit par le déphasage entre le front d'onde qui traverse la cape et celui qui se propage en espace libre.

Des capes d'invisibilité pour la protection contre les ondes délétères: Faisons maintenant l'expérience de pensée suivante : imaginons que nous sommes un observateur à la surface de l'eau. Que se passe-t-il quand nous sommes situés dans la région centrale du dispositif dans la figure 1 id est dans la zone d'invisibilité de la cape à vagues ? Nous sommes coupés du monde extérieur, nous vivons en quelque sorte dans un monde parallèle qui nous est propre. Contrairement au personnage de fiction Harry Potter, nous ne savons rien de ce qui se passe à la surface de l'eau en dehors de la cape à vagues. Si nous étions Harry, nous serions dans le noir le plus complet, et s'il nous prenait l'envie d'allumer une torche, nous serions éblouis par une lumière très intense : la cape agit comme une cavité parfaite, aucune onde lumineuse n'en sort en théorie. En définitive, aucune onde lumineuse ne pénètre ou ne sort de la région centrale de la cape d'invisibilité de Harry, et il en est de même pour les vagues. La cape à vague mise à l'échelle métrique peut dès lors servir à protéger des plateformes offshores des vagues délétères en pleine mer. Des capes d'invisibilité ont même été proposées pour les ondes sismiques, dont la structure vue du ciel ressemble fortement à celle de la cape à vagues en figure 1 [ref2], à ceci près que les pylônes constituant la cape sont maintenant des colonnes de béton savamment agencées dans le sol ou des arbres plantés judicieusement sur le sol.

Des tamis à photons pour la transparence de métaux avec des petits trous : La médaille d'or du CNRS en 2019 vient récompenser les travaux du physico-chimiste franco-norvégien Thomas Ebbesen dans le domaine dit de l'optique plasmonique. Ebbesen et son équipe strasbourgeoise ont découvert il y a une vingtaine d'années que des photons passent à travers des trous minuscules dans des parois métalliques à l'instar de "l'excellent homme nommé Dutilleul qui possédait le don singulier de passer à travers les murs sans en être incommodé » dans le Passe-muraille de Marcel Aymé. Ce tour de passepasse est réalisé par le couplage de la lumière avec des plasmons dits chafouins (traduction littérale de spoof-plasmons dans la langue de Shakespeare) qui sont des déplacements collectifs d'électrons à la surface du métal. Les plasmons chafouins sont l'analogue des vagues à la surface de la mer et des ondes mécaniques de Rayleigh se propageant à l'interface air-sol durant les séismes.

[ref1]Sébastien Guenneau et Boris Gralak. “ Une optique classique sens dessus dessous", Les Dossiers de la Recherche, Février 2010, p.32-36 [ref2]Sébastien Guenneau, Stefan Enoch, Andrea Colombi, Philippe Roux et Stéphane Brûlé. "Métamatériaux pour la protection sismiques", Photoniques, Janvier 2018, p.37-40 
Thomas Ebbesen parle de transmission extraordinaire à travers des tamis à photons pour signifier que le métal devient transparent comme le verre pour certaines fréquences (ou couleurs) de la lumière visible, mais reste opaque pour les autres fréquences (ou couleurs). Ces tamis à photons agissent donc comme des filtres à lumière. Des filtres analogues ont été proposés pour les vagues afin de régulariser la houle se propageant à travers des digues qui filtrent les bonnes et mauvaises vagues, afin de réduire les phénomènes d'érosion côtière. Des tamis à ondes de Rayleigh ont été proposés avec des forêts d'arbres, qui filtrent les différences fréquences des séismes pour les rendre moins délétères.

Conclusion : Le spectre électromagnétique est large depuis les petits rayons $X$ de longueur d'onde inférieure au nanomètre jusqu'aux grandes ondes radio de plusieurs mètres et si une cape d'invisibilité détourne certaines fréquences d'ondes électromagnétiques, elle se fera traverser par les autres ou vice versa. Tout dépend de l'épaisseur de la cape et de la taille de ses petits éléments structurels : il faut que les longueurs d'onde considérées excitent les résonances internes des petits éléments, et il faut de plus que la cape soit assez épaisse pour jouer son rôle. En conséquence, le personnage sous la cape d'invisibilité sera invisible pour un spectre de fréquences étroit et visible pour les autres longueurs d'ondes. Mais en retour il pourra pressentir certains aspects du monde extérieur, à l'instar des hommes de la caverne de Platon. De même, un tamis à photons qui consiste à percer un métal avec des trous minuscules pour le rendre transparent comme le verre à certaines fréquences d'ondes lumineuses, mais reste opaque aux autres fréquences. Etonnamment, les concepts d'invisibilité et de transparence trouvent leurs premières applications pratiques dans les domaines de la protection contre les vagues et les séismes. En effet, la réalisation d'une cape d'invisibilité pour contrôler la lumière du visible est à l'heure actuelle hors de portée des techniques de fabrication les plus avancées en nanotechnologie, car elle nécessite de structurer très finement la matière à l'échelle de quelques nanomètres. Par contre, la fabrication de capes de protection contre les vagues et les séismes fait appel à des techniques de construction bien rôdées en génies civil et maritime.

\section{Biographie courte des auteurs :}

Boris Gralak est chercheur au CNRS à l'Institut Fresnel à Marseille depuis 2004. Il est spécialiste en modélisation électromagnétique et en modélisation des métamatériaux. II a soutenu sa thèse sur l'étude théorique et numérique des cristaux photoniques à l'université d'Aix-Marseille en 2001. De 2001 à 2004, il a effectué ses recherches au FOM-Institute [Amolf] à Amsterdam.

Sébastien Guenneau est chercheur au CNRS depuis 2006. II est spécialiste de la physique des métamatériaux pour les ondes mécaniques. II a soutenu sa thèse sur la modélisation des fibres optiques micro-structurées et les propriétés effectives des quasi-cristaux à l'Université d'Aix-Marseille en 2001. Ses travaux postdoctoraux ont été effectués à Liverpool, Londres et Marseille. 